\title{
Causality implies inflationary back-reaction
}

\section{S. Basu, ${ }^{a}$ N.C. Tsamis $^{b}$ and R.P. Woodard ${ }^{a}$}

${ }^{a}$ Department of Physics, University of Florida, Gainesville, FL 32611, U.S.A.

${ }^{b}$ Institute of Theoretical Physics $\mathcal{E}$ Computational Physics, Department of Physics, University of Crete, GR-710 03 Heraklion, Greece

E-mail: shinjinibasu@ufl.edu, tsamis@physics.uoc.gr, woodard@phys.ufl.edu

ABSTRACT: There is a widespread belief among inflationary cosmologists that a local observer cannot sense super-horizon gravitons. The argument goes that a local observer would subsume super-horizon gravitons into a redefinition of his coordinate system. We show that adopting this view for pure gravity on de Sitter background leads to time variation in the Hubble parameter measured by a local observer. It also leads to a violation of the gravitational field equation $R=4 \Lambda$ because that equation is obeyed by the full metric, rather than the one which has been cleansed of super-horizon modes.

KEYWORDs: Cosmology of Theories beyond the SM, Models of Quantum Gravity

ARXIV EPRINT: 1612.07406 


\section{Contents}

1 Introduction 1

2 The transformation $\quad 2$

3 Back-reaction 3

3.1 The graviton expansion of $\mathrm{H}(x) \quad 4$

$\begin{array}{ll}3.2 & \text { The coincident graviton propagator }\end{array}$

$\begin{array}{lll}3.3 & \text { IR cleansed Hubble parameter \& field equation } & 7\end{array}$

$\begin{array}{llr}4 & \text { Epilogue } & 9\end{array}$

\section{Introduction}

One of the peculiar features of quantum field theory during inflation is the existence of secular corrections from loops of massless, minimally coupled scalars [1-13] and/or gravitons [14-23]. These corrections grow without bound as long as inflation lasts. Among the many interesting effects caused by these secular corrections are changes to particle kinematics [24-32], changes in long range forces [18, 19, 33-35], changes in the primordial power spectra $[10,36,37]$, and changes in the cosmic expansion rate [5, 38-40].

There is no dispute about the reality of secular corrections driven by massless, minimally coupled scalars. The stochastic formalism of Starobinsky [41] even provides a method for working out what happens at late times in those cases which approach a static limit [5, 40, 42-46]. On the other hand, there are fierce debates concerning both the reality of secular corrections from inflationary gravitons and what they might do after perturbation theory breaks down $[47,48]$.

Secular effects derive from more and more of the plane wave mode functions of free scalar and graviton fields approaching a nonzero, spacetime constant [49-51]. On de Sitter background both mode functions are ${ }^{1}$

$$
u(\eta, k)=\frac{H}{\sqrt{2 k^{3}}}\left[1-\frac{i k}{H a(\eta)}\right] \exp \left[\frac{i k}{H a(\eta)}\right] \rightarrow \frac{H}{\sqrt{2 k^{3}}}, \quad a(\eta)=-\frac{1}{H \eta} .
$$

Their approach to a constant is known as "freezing in" and it is how inflationary perturbations survive to much later times. Physicists accept that the freezing in of scalars can mediate effects because the value of a scalar field is observable; for example, the expectation value of the Higgs field determines masses in the Standard Model. However, physicists are conditioned to dismiss nonzero constant values of the graviton field as gauge artifacts which could be eliminated by an appropriate choice of coordinates [52-57].

\footnotetext{
${ }^{1}$ The small $k$ and late time limiting forms of $u(\eta, k)$ are the same, which has led to much confusion between infrared divergences and secular growth. The former derive from the region near $k=0$, while secular growth derives from the region near $k=H a(\eta)$, which grows without bound.
} 
This belief is problematic because the graviton mode functions are not exactly constant. They vary rapidly at early times $(k \gg H a)$, and it was never clear why their passage to the late time regime $(k \ll H a)$ can have no observable effect beyond the tensor power spectrum [58]. However, a supporting argument is adduced based on the presumed difficulty of a local observer in resolving the spacetime variation of any mode whose wavelength exceeds the causal horizon $(k<H a(\eta))[59,60]$. It is asserted that a local observer would instead subsume these super-horizon modes into a transformation of his coordinate system [61-64]. We refer to this belief as the Transformation Ansatz [65].

There are several reasons to doubt the Transformation Ansatz. First, field theory interactions are local in spacetime, not in Fourier space. Variations of long wavelength modes are indeed difficult to resolve, but this is a consequence of local dynamics and does not require the excision of dynamical variables by some deux ex machina. Second, the range of excluded $k$ values changes with time: it increases during inflation and decreases after the end of inflation. Finally, the fact that inflation ends means that the Hubble radius is not a true horizon, nor is there any invariant meaning to wave number $k$ in the full, interacting theory.

Yet the Transformation Ansatz has many adherents [66-72], and it is invoked to deny the possibility of secular graviton corrections in general, and of secular back-reaction in particular. Our purpose here is not to pass on the validity of the Transformation Ansatz but rather to demonstrate that adopting it leads to precisely the opposite conclusion about secular back-reaction. The reason should become clear when we carefully examine what happens to the conformal factor upon attempting to absorb super-horizon gravitons into a redefinition of coordinates. If $h_{\mu \nu}(x)$ represents the graviton field that is approaching a constant, the actual metric is not $\eta_{\mu \nu}+\kappa h_{\mu \nu}(x)$ - which really would be trivial if constant - but rather,

$$
g_{\mu \nu}(x)=a^{2}\left[\eta_{\mu \nu}+\kappa h_{\mu \nu}\right] \equiv a^{2} \widetilde{g}_{\mu \nu} \quad \kappa^{2} \equiv 16 \pi G .
$$

The coordinate transformation which carries $\widetilde{g}_{\mu \nu}$ to $\eta_{\mu \nu}$ - under the false assumption that $\widetilde{g}_{\mu \nu}$ is exactly constant - changes how the scale factor depends upon the new time coordinate. This leads to a secular decrease of the Hubble parameter and a violation of the field equation.

In section 2 we give the transformation as a function of the graviton field, assuming (according to the Transformation Ansatz) that it is exactly constant. In section 3 we show that the expected Hubble parameter decreases at order $\kappa^{4}$, and that there is a corresponding violation of the Einstein equation. Section 4 discusses the fascinating question of what this all might mean.

\section{The transformation}

We deal with three different metrics:

- The true metric $g_{\mu \nu}(x)$, which includes the scale factor and super-horizon modes;

- The conformally rescaled metric $\widetilde{g}_{\mu \nu}(x) \equiv g_{\mu \nu}(x) / a^{2}$ which has the conformal factors cancelled but still contains super-horizon modes; and

- The local observer's metric $\mathbf{g}_{\mu \nu}(x)$ which has been cleansed of super-horizon modes by subsuming them into a coordinate redefinition. 
We also decompose the conformally rescaled metric $\widetilde{g}_{\mu \nu}(x)$ into a super-horizon part $\gamma_{\mu \nu}(x)=\eta_{\mu \nu}+\kappa \psi_{\mu \nu}(x)$, and a sub-horizon part $\kappa \chi_{\mu \nu}(x)$,

$$
\widetilde{g}_{\mu \nu}(x) \equiv \gamma_{\mu \nu}(x)+\kappa \chi_{\mu \nu}(x) \text {. }
$$

The local observer's metric is defined by constructing the linear coordinate transformation $x^{\mu} \rightarrow x^{\prime \mu}$ which would carry $\gamma_{\mu \nu}(x)$ to $\eta_{\mu \nu}$ under the Transformation Ansatz assumption that $\gamma_{\mu \nu}(x)$ is exactly constant. We use the matrix coefficients $\omega^{\mu}{ }_{\nu}$ to denote the inverse transformation $x^{\mu}=\omega_{\nu}^{\mu} x^{\prime \nu}$. The local observer's metric is,

$$
\mathbf{g}_{\mu \nu}(x) \equiv \omega^{\rho}{ }_{\mu} \omega^{\sigma}{ }_{\nu} g_{\rho \sigma}(\omega x)=a^{2}\left(\omega^{0}{ }_{\alpha} x^{\alpha}\right) \times\left[\eta_{\mu \nu}+\omega^{\rho}{ }_{\mu} \omega^{\sigma}{ }_{\nu} \kappa \chi_{\rho \sigma}(\omega x)\right] .
$$

In addition to having the property $\omega^{\rho}{ }_{\mu} \omega_{\nu}^{\sigma} \gamma_{\mu \nu}(x)=\eta_{\mu \nu}$ we want the local observer's scale factor to depend only on conformal time, which means $\omega_{i}^{0}=0$. The solution for $\omega^{\mu}{ }_{\nu}$ turns out to be the Lorentz-symmetric vierbein [73] with a Lorentz boost to null the time-space components [65],

$$
\omega_{\nu}^{\mu} \equiv\left(\begin{array}{cc}
\omega_{0}^{0} & \omega_{n}^{0} \\
\omega_{0}^{m} & \omega_{n}^{m}
\end{array}\right)=\left(\begin{array}{cc}
\frac{1}{N} & 0 \\
\frac{N^{m}}{N} & e_{n}^{m}
\end{array}\right),
$$

where $N$ and $N^{m}$ are the lapse and shift [74-76] of $\gamma_{\mu \nu}$, and $e_{n}^{m}$ is the inverse driebein of its spatial components $\gamma_{m n}=\Gamma_{m n}$ (i.e., $\Gamma^{m n}=e_{k}^{m} e_{k}^{n}$ ),

$$
\frac{1}{N}=\sqrt{-\gamma^{00}}, \quad \frac{N^{m}}{N}=-\frac{\gamma^{0 m}}{\sqrt{-\gamma^{00}}}, \quad e_{n}^{m}=\left(\sqrt{\Gamma^{-1} \times I}\right)_{n}^{m} .
$$

The transformation (2.3)-(2.4) is unique up to a 3-rotation of the inverse driebein, $e_{n}^{m} \rightarrow e_{k}^{m} \times R_{k n}$, which plays no role for us.

We should emphasize that $x^{\mu}=\omega^{\mu}{ }_{\nu} x^{\prime \nu}$ is not a true coordinate transformation because the matrix $\omega^{\mu}{ }_{\nu}$ given by $(2.3)-(2.4)$ is not a spacetime constant,

$$
\partial_{\rho} \omega_{\sigma}^{\mu} \neq \partial_{\sigma} \omega_{\rho}^{\mu} \quad \Longrightarrow \quad \omega_{\nu}^{\mu} \neq \frac{\partial x^{\mu}}{\partial x^{\prime \nu}} .
$$

The principle obstacle in (2.5) is not the small residual spacetime dependence of any particular super-horizon mode in $\psi_{\mu \nu}(x)$ but rather the fact that more and more modes make the transition from $\chi_{\mu \nu}(x)$ to $\psi_{\mu \nu}(x)$ as they experience horizon crossing. This means we must view the local observer's metric (2.2) as a nonlocal field redefinition of the original metric, which may not be expanding at the same rate and may not even obey the same local field equation.

\section{Back-reaction}

Expressions (2.2) and (2.3)-(2.4) imply that the local observer's scale factor is,

$$
a\left(\omega_{\rho}^{0} x^{\rho}\right)=-\frac{1}{H \sqrt{-\gamma^{00}} \times \eta} .
$$


This is de Sitter with a rescaled Hubble constant which depends slightly on spacetime through the super-horizon part of the graviton field,

$$
\mathbf{H}(x) \equiv H \times \sqrt{-\gamma^{00}(x)} .
$$

The residual time dependence of super-horizon mode functions is not significant but the continual addition of new modes - as they pass from the sub-horizon graviton field $\chi_{\mu \nu}(x)$ to the super-horizon graviton field $\psi_{\mu \nu}(x)$ - introduces an appreciable time dependence. We begin by expanding the operator $\mathbf{H}(x)$ in powers of $\psi_{\mu \nu}$. We then compute the expectation value at 4 th order, and the section closes with a discussion of the field equation obeyed by the local observer's metric $\mathbf{g}_{\mu \nu}(x)$.

One might wonder about the effect of modes which are super-horizon even on the initial value surface. This is a fascinating question whose answer we do not know. However, there is a simple way to distinguish these initially super-horizon modes from initially sub-horizon modes which experience first horizon crossing during the course of inflation. This is just to work on a finite spatial manifold such as $T^{3}$, which supports spatially flat de Sitter background. If the initial physical radius of the manifold is smaller than the Hubble length then there are no initially super-horizon modes. This is also a standard technique for controlling infrared divergences [77].

\subsection{The graviton expansion of $\mathbf{H}(x)$}

Inverting $\gamma_{\mu \nu}(x)=\eta_{\mu \nu}+\kappa \psi_{\mu \nu}$, employing the usual convention about raising and lowering graviton indices with $\eta_{\mu \nu}$, and taking account of the spacelike signature gives,

$$
\gamma^{00}=-1+\kappa \psi_{00}-\kappa^{2} \psi_{0}{ }^{\rho} \psi_{\rho 0}+\kappa^{3} \psi_{0 \rho} \psi_{\sigma}^{\rho} \psi_{0}^{\sigma}-\kappa^{4} \psi_{0 \rho} \psi_{\sigma}^{\rho} \psi_{\tau}^{\sigma} \psi_{0}^{\tau}+O\left(\kappa^{5}\right)
$$

Substituting in expression (3.2) results in the expansion,

$$
\begin{aligned}
\frac{\mathbf{H}(x)}{H}= & \frac{1}{N(x)}=\sqrt{-\gamma^{00}(x)} \\
= & 1+\frac{\kappa}{2} \psi_{00}-\frac{\kappa^{2}}{2}\left[\psi_{0 \rho} \psi_{0}^{\rho}+\frac{1}{4} \psi_{00}^{2}\right]+\frac{\kappa^{3}}{2}\left[\psi_{0 \rho} \psi_{\sigma_{\sigma}}^{\rho} \psi_{0}^{\sigma}+\frac{1}{2} \psi_{0 \rho} \psi_{0}^{\rho} \psi_{00}+\frac{1}{8} \psi_{00}^{3}\right] \\
& -\frac{\kappa^{4}}{2}\left[\psi_{0 \rho} \psi_{{ }_{\sigma}}^{\rho} \psi_{{ }_{\tau}}^{\sigma} \psi_{0}^{\tau}+\frac{1}{4}\left(\psi_{0 \rho} \psi_{0}^{\rho}\right)^{2}+\frac{1}{2} \psi_{0 \rho} \psi^{\rho}{ }_{\sigma} \psi_{0}{ }_{0} \psi_{00}\right. \\
& \left.+\frac{3}{8} \psi_{0 \rho} \psi_{0}^{\rho}{ }_{0} \psi_{00}^{2}+\frac{5}{64} \psi_{00}^{4}\right]+O\left(\kappa^{5}\right) .
\end{aligned}
$$

Expression (3.5) is a series of quantum operators. Even after horizon crossing these operators are superpositions of random numbers, so we can only discuss the statistical properties of $\mathbf{H}(x)$ rather than its numerical value. Taking the expectation value gives a series of diagrams having the general form shown in figure 1. Basically, any term in expression (3.5) which contains $N$ factors of the super-horizon graviton field $\psi_{\mu \nu}(x)$ corresponds to a diagram with $N$ lines emanating from the top vertex and then joining with all possible interactions. For example, figure 2 shows the expansion for $N=2$. 


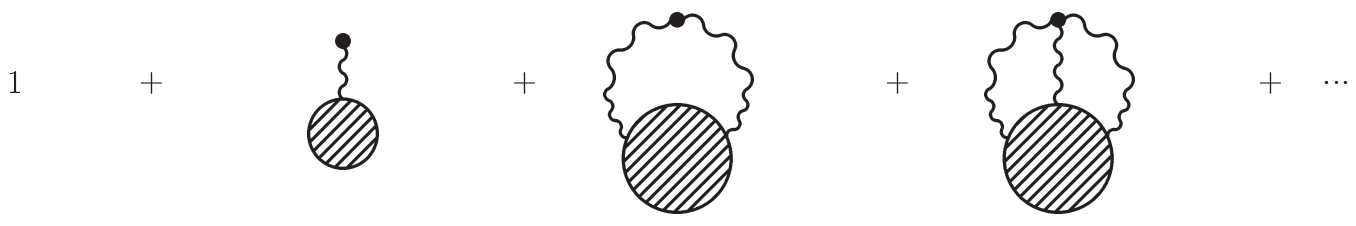

Figure 1. Expanding $\mathrm{H}(x) / H$ in powers of the graviton field and then taking the expectation value results in the diagrammatic series depicted above. The spacetime point $x^{\mu}$ is the solid vertex.
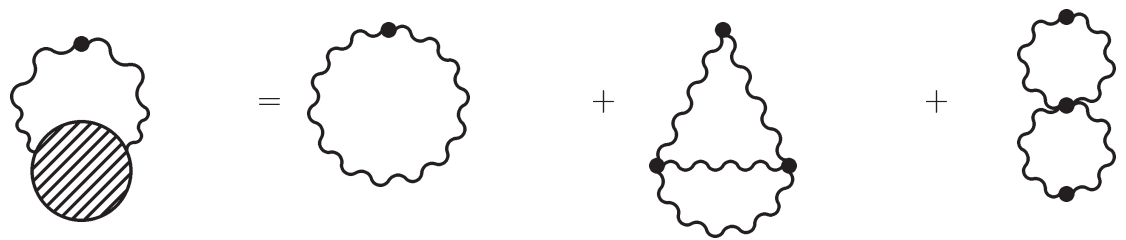

Figure 2. Each of the $N$-point functions of figure 1 has a conventional diagrammatic expansion. This graph shows the lowest terms which contribute to the 2-point function.

To understand the diagrams in figures 1 and 2 quantitatively it is important to go beyond linearized order in the free field expansion, and to be precise about what we mean by the sub-horizon and super-horizon parts. The Heisenberg field equations of general relativity permit us to express the full graviton field $h_{\mu \nu}(x)$ as a series in powers of the linearized solutions $h_{\mu \nu}^{(1)}(x)$ about de Sitter background,

$$
h_{\mu \nu}(x)=h_{\mu \nu}^{(1)}(x)+\kappa h_{\mu \nu}^{(2)}(x)+\kappa^{2} h_{\mu \nu}^{(3)}(x)+\ldots
$$

Here $h_{\mu \nu}^{(m)}$ represents the term in the full solution which contains $m$ factors of the free field $h_{\mu \nu}^{(1)}$, generally integrated against and contracted into vertices,

$$
\begin{aligned}
h_{\mu \nu}^{(m)}(x)= & \int d^{D} x_{1} h_{\alpha_{1} \beta_{1}}^{(1)}\left(x_{1}\right) \cdots \\
& \times \int d^{D} x_{m} h_{\alpha_{m} \beta_{m}}^{(1)}\left(x_{m}\right) \times \mathcal{V}_{\mu \nu}{ }^{\alpha_{1} \beta_{1} \cdots \alpha_{m} \beta_{m}}\left(x ; x_{1}, \ldots, x_{m}\right) .
\end{aligned}
$$

It is only the free field $h_{\mu \nu}^{(1)}=\chi_{\mu \nu}^{(1)}+\psi_{\mu \nu}^{(1)}$ which can be simply decomposed into subhorizon and super-horizon parts. To apportion the higher order contributions we adopt the principle of Contagion, whereby the presence of even a single factor of $\psi_{\mu \nu}^{(1)}$ renders the entire term "super-horizon". So substituting $h_{\mu \nu}^{(1)}=\chi_{\mu \nu}^{(1)}+\psi_{\mu \nu}^{(1)}$ in the $m$ free fields of expression (3.7) results in a single contribution to $\chi_{\mu \nu}^{(m)}$ from $\chi_{\alpha_{1} \beta_{1}}^{(1)}\left(x_{1}\right) \cdots \chi_{\alpha_{m} \beta_{m}}^{(1)}\left(x_{m}\right)$ and $2^{m}-1$ contributions to $\psi_{\mu \nu}^{(m)}$.

So the graphs represented in figures 1 and 2 are not quite conventional Feynman diagrams. Because we are taking the expectation value of an operator (3.5) which depends only on the long wavelength graviton field $\psi_{\mu \nu}(x)$, the lines emanating from the top point $x^{\mu}$ are only the long wavelength $(k<H a(\eta))$ part of the free propagator mode sums. By Contagion, all the internal vertices and propagators are those of the full theory. 
It should not be surprising, and is confirmed by explicit computation [78], that secular enhancements derive entirely from the purely spatial parts of the free graviton field $\psi_{i j}^{(1)}$. Gravitons with both indices temporal $\left(\psi_{00}^{(1)}\right)$, or with mixed time and space indices $\left(\psi_{0 i}^{(1)}\right)$ make nonzero contributions, but these contributions do not grow with time. This means we can make a great reduction in the expectation value of the order $\kappa^{4}$ terms in (3.5),

$$
\begin{gathered}
-\frac{\kappa^{4}}{2}\left\langle\Omega\left|\psi_{0 \rho} \psi_{\sigma}^{\rho} \psi_{\tau}^{\sigma} \psi_{0}^{\tau}+\frac{1}{4}\left(\psi_{0 \rho} \psi_{0}^{\rho}\right)^{2}+\frac{1}{2} \psi_{0 \rho} \psi_{\sigma}^{\rho} \psi_{0}^{\sigma} \psi_{00}+\frac{3}{8} \psi_{0 \rho} \psi_{0}^{\rho} \psi_{00}^{2}+\frac{5}{64} \psi_{00}^{4}\right| \Omega\right\rangle \\
\longrightarrow-\frac{\kappa^{4}}{2}\left\langle\Omega\left|\psi_{0 i}^{(1)}(x) \psi_{0 j}^{(1)}(x)\right| \Omega\right\rangle\left\langle\Omega\left|\psi_{i k}^{(1)}(x) \psi_{j k}^{(1)}(x)\right| \Omega\right\rangle+O\left(\kappa^{6}\right)
\end{gathered}
$$

The order $\kappa^{4}$ part of (3.8) consists of coincident propagators whose evaluation we now discuss.

\subsection{The coincident graviton propagator}

We control ultraviolet divergences with dimensional regularization in spacetime dimension $D$. Almost all graviton loops on de Sitter have been computed using a noncovariant gauge fixing term $[79,80]$,

$$
\mathcal{L}_{\mathrm{GF}}=-\frac{1}{2} a^{D-2} \eta^{\mu \nu} F_{\mu} F_{\nu}, \quad F_{\mu} \equiv \eta^{\rho \sigma}\left[h_{\mu \rho, \sigma}-\frac{1}{2} h_{\rho \sigma, \mu}+(D-2) a H h^{\mu \rho} \delta_{\sigma}^{0}\right] .
$$

The resulting propagator is a sum of three products of a scalar propagator times a constant tensor factor $[79,80]$,

$$
i\left[{ }_{\mu \nu} \Delta_{\rho \sigma}\right]\left(x ; x^{\prime}\right)=\sum_{I=A, B, C} i \Delta_{I}\left(x ; x^{\prime}\right) \times\left[\mu \nu T_{\rho \sigma}^{I}\right] .
$$

The propagators are those for a scalar of masses $m_{A}^{2}=0, m_{B}^{2}=(D-2) H^{2}$ and $m_{C}^{2}=2(D-3) H^{2}$. The tensor factors are constructed from $\delta_{\mu}^{0}$ and the spatial part of the Lorentz metric $\bar{\eta}_{\mu \nu} \equiv \eta_{\mu \nu}+\delta_{\mu}^{0} \delta_{\nu}^{0}$

$$
\begin{aligned}
& {\left[{ }_{\mu \nu} T_{\rho \sigma}^{A}\right]=2 \bar{\eta}_{\mu(\rho} \bar{\eta}_{\sigma) \nu}-\frac{2}{D-3} \bar{\eta}_{\mu \nu} \bar{\eta}_{\rho \sigma},} \\
& {\left[{ }_{\mu \nu} T_{\rho \sigma}^{B}\right]=-4 \delta_{(\mu}^{0} \bar{\eta}_{\nu)\left(\rho^{\prime} \delta_{\sigma}^{0},\right.}} \\
& {\left[{ }_{\mu \nu} T_{\rho \sigma}^{C}\right]=\frac{2}{(D-2)(D-3)}\left[(D-3) \delta_{\mu}^{0} \delta_{\nu}^{0}+\bar{\eta}_{\mu \nu}\right]\left[(D-3) \delta_{\rho}^{0} \delta_{\sigma}^{0}+\bar{\eta}_{\rho \sigma}\right] .}
\end{aligned}
$$

Note that parenthesized indices are symmetrized.

The full spacetime dependence of all three scalar propagators is known $[79,80]$ but we only require their coincidence limits. Secular growth comes entirely from the $A$-type propagator [49-51],

$$
i \Delta_{A}(x ; x)=\text { Constant }+\frac{H^{2}}{4 \pi^{2}} \ln (a) .
$$


At coincidence the $B$-type and $C$-type propagators are actually finite in dimensional regularization [80],

$$
\begin{aligned}
& i \Delta_{B}(x ; x)=-\frac{H^{D-2}}{(4 \pi)^{\frac{D}{2}}} \times \frac{\Gamma(D-2)}{\Gamma\left(\frac{D}{2}\right)} \longrightarrow-\frac{H^{2}}{16 \pi^{2}}, \\
& i \Delta_{C}(x ; x)=+\frac{H^{D-2}}{(4 \pi)^{\frac{D}{2}}} \times \frac{\Gamma(D-3)}{\Gamma\left(\frac{D}{2}\right)} \longrightarrow+\frac{H^{2}}{16 \pi^{2}} .
\end{aligned}
$$

The time dependent part of (3.14) comes entirely from the super-horizon contributions to the mode sum of the A-type propagator. In contrast, the nonzero constants of (3.15)(3.16) derive from sub-horizon as well as super-horizon modes. However, because the super-horizon mode sum runs from $k=0$ to $k=H a(\eta) \rightarrow \infty$, we make only a small error (and one which falls off with time) in regarding expressions (3.15)-(3.16) as the expectation values of just the super-horizon mode sums. Hence we can evaluate (3.8) as,

$$
\begin{aligned}
- & \frac{\kappa^{4}}{2}\left\langle\Omega\left|\psi_{0 \rho} \psi_{\sigma}^{\rho} \psi_{\tau}^{\sigma} \psi_{0}^{\tau}+\frac{1}{4}\left(\psi_{0 \rho} \psi_{0}^{\rho}\right)^{2}+\frac{1}{2} \psi_{0 \rho} \psi_{\sigma}^{\rho} \psi_{0}^{\sigma} \psi_{00}+\frac{3}{8} \psi_{0 \rho} \psi_{0}^{\rho} \psi_{00}^{2}+\frac{5}{64} \psi_{00}^{4}\right| \Omega\right\rangle \\
& \left.\longrightarrow-\frac{\kappa^{4}}{2} \times i\left[{ }_{0 i} \Delta_{0 j}\right](x ; x) \times i{ }_{i k} \Delta_{j k}\right](x ; x)+O\left(\kappa^{6}\right) \\
= & -\frac{\kappa^{4}}{2} \times-\delta_{i j} i \Delta_{B}(x ; x) \times\left(D-\frac{2}{D-3}\right) \delta_{i j} i \Delta_{A}(x ; x)+O\left(\kappa^{6}\right) \\
& \longrightarrow-\frac{3(\kappa H)^{4}}{64 \pi^{4}} \ln (a)+O\left(\kappa^{6}\right) .
\end{aligned}
$$

Although the initial expansion (3.5) of $\mathbf{H} / H$ in powers of the graviton field is the same in all gauges, the evaluation of the expectation value of individual terms in this expansion, such as (3.8), is of course dependent upon the gauge. We have chosen to work in the covariant gauge (3.9), which is the simplest to use. However, it is worth describing how the same effect would appear in the much more complicated formalism associated with a physical gauge such as $\partial_{i} \psi_{i j}=0=\psi_{i i}$. In that gauge the spatial-transverse-traceless components of the graviton field $\psi_{i j}^{T T}$ are invariant under linearized gauge transformations, although they are not fully invariant. The other components of the metric, including $\psi_{00}$ and $\psi_{0 i}$, are also not zero. They would be expressed as series expansions in powers of $\psi_{i j}^{T T}$, staring at order $\kappa \psi^{2}$, by perturbatively solving the constraint equations. So the same order $\kappa^{4}$ effect that we obtained in expression (3.19) might derive from the expectation value of the terms $\kappa^{2}\left[\frac{3}{8} \psi_{00}^{2}-\frac{1}{2} \psi_{0 i} \psi_{0 i}\right]$ from expression (3.5). Note that the gauge-fixed and constrained Lagrangian of a physical gauge is not local, and can of course only be expressed to some finite order because exact solutions for the constraint equations are not known. That is what makes this formalism so terrifically difficult to use. In fact all graviton loop computations on de Sitter background have been performed using covariant gauges for which the Lagrangian is local and the graviton propagator includes both constrained and physical components.

\subsection{IR cleansed Hubble parameter \& field equation}

Recall that expression (3.5) for $\mathbf{H}(x) / H$ contains terms with $N$ factors of $\psi_{\mu \nu}$ for $N=0,1,2, \ldots$ The expectation value has constant contributions at order $\kappa^{2}$ from the 
$N=1$ [81] and $N=2$ terms, which can be absorbed into a renormalization of the cosmological constant. Expression (3.19) gives the secular contribution from the $N=4$ term. If we assume there are no secular contributions at order $\kappa^{4}$ from the $N=1, N=2$ and $N=3$ terms (more on this later) then the expectation value of $\mathbf{H}(x)$ is,

$$
\langle\Omega|\mathbf{H}(x)| \Omega\rangle \longrightarrow H\left\{1-\frac{3(\kappa H)^{4}}{64 \pi^{4}} \ln (a)+O\left(\kappa^{6}\right)\right\} .
$$

It is interesting to note that secular slowing is predicted to occur at the same order, and with the same time dependence, when one does not excise the super-horizon modes but rather includes their contribution to the vacuum energy [82].

It is worth digressing at this point to note that our result (3.20) applies even to pure quantum gravity, with a positive cosmological constant, released in Bunch-Davies vacuum. One consequence is that gravitons do not possess a fully de Sitter invariant vacuum state, just like the massless, minimally coupled scalar [83], whose plane wave mode functions are identical to those of dynamical gravitons [84]. There has been a long and confusing debate about this [52-57]. All agree that the graviton mode functions approach a constant at late times (that is what causes the tensor power spectrum) and that this freezing-in endows the completely gauge fixed graviton propagator with a de Sitter-breaking time dependence which takes the form of a linearized gauge transformation. The debate concerns whether or not this time dependence can have physical consequences analogous to those of the constant gauge field in the famous Aharonov-Bohm effect [85]. Our attitude is to decide the matter by computation, using the propagator described in section 3.2 which all sides accept as valid. Our result (3.20) does support the view that de Sitter breaking is real, although this conclusion needs to be confirmed by a complete, two loop computation of an invariant measure of the local expansion rate [86].

A final comment concerns the magnitude and universality of the effect. Even during primordial inflation, the dimensionless loop counting parameter is minuscule, $\kappa^{2} H^{2}<10^{-10}$. However, the factor of $\ln (a)$ grows with time so that the effect must eventually become nonperturbatively strong. Expression (3.20) was derived using perturbation theory, hence it is valid so long as $\kappa^{2} H^{2} \ln (a)$ is small. This means that it applies to the early stages of inflation for any vacuum energy of a few orders of magnitude below the Planck mass, all the way down to zero.

The original metric $g_{\mu \nu}(x)$ obeys the exact Heisenberg field equation $R(x)=D \Lambda$, where the cosmological constant $\Lambda$ is $(D-1) H^{2}$ plus renormalization counterterms. That fact has been invoked to claim that there can be no back-reaction [47, 48]. However, it could be argued that one must instead re-organize the operators of the Ricci scalar so as to extract quantum corrections to the vacuum energy, the same way one does for the generator $L_{0}$ in Virasoro algebra of free string theory [87]. In that case back-reaction would derive from integrating the vertices of loop corrections back to the initial value surface, over the larger and larger past light-cones which open as the observation point $x^{\mu}$ occurs later and later after the initial value surface. Although the process is completely causal in spacetime, it does involve contributions from the super-horizon modes $\psi_{\mu \nu}$ of the graviton field, which 
disturbs those who believe in the Transformation Ansatz. We therefore examine the field equation obeyed by the local observer's metric (2.2) which is free of super-horizon modes.

It is useful to extract the local observer's scale factor (3.1) from his metric (2.2),

$$
\mathbf{g}_{\mu \nu}(x) \equiv \mathbf{a}^{2}(x) \times \widehat{\mathbf{g}}_{\mu \nu}(x), \quad \mathbf{a}(x) \equiv-\frac{1}{\mathbf{H}(x) \eta} .
$$

The local observer's Ricci tensor follows from a conformal transformation,

$$
\begin{aligned}
\mathbf{R}_{\mu \nu}= & \widehat{\mathbf{R}}_{\mu \nu}-(D-2)\left(\frac{\mathbf{a}_{, \mu}}{\mathbf{a}}\right)_{; \nu}-\widehat{\mathbf{g}}_{\mu \nu} \widehat{\mathbf{g}}^{\rho \sigma}\left(\frac{\mathbf{a}_{, \rho}}{\mathbf{a}}\right)_{; \sigma} \\
& +(D-2) \frac{\mathbf{a}_{, \mu}}{\mathbf{a}} \frac{\mathbf{a}_{, \nu}}{\mathbf{a}}-(D-2) \widehat{\mathbf{g}}_{\mu \nu} \widehat{\mathbf{g}}^{\rho \sigma} \frac{\mathbf{a}_{, \rho}}{\mathbf{a}} \frac{\mathbf{a}_{, \sigma}}{\mathbf{a}},
\end{aligned}
$$

where a comma denotes ordinary differentiation and a semicolon indicates covariant differentiation with the affine connection of $\widehat{\mathbf{g}}_{\mu \nu}$. If we ignore the small spacetime variation of $\mathbf{H}(x)$ then derivatives of the scale factor are,

$$
\begin{aligned}
\frac{\mathbf{a}, \mu}{\mathbf{a}} & \longrightarrow-\frac{\delta^{0}{ }_{\mu}}{\eta}=\mathbf{H a} \delta^{0}{ }_{\mu}, \\
\left(\frac{\mathbf{a}_{, \mu}}{\mathbf{a}}\right)_{; \nu} & \longrightarrow \mathbf{H}^{2} \mathbf{a}^{2} \delta^{0}{ }_{\mu} \delta^{0}{ }_{\nu}-\widehat{\boldsymbol{\Gamma}}^{0}{ }_{\nu \mu} \mathbf{H a} \longrightarrow \mathbf{H}^{2} \mathbf{a}^{2} \delta^{0}{ }_{\mu} \delta^{0}{ }_{\nu},
\end{aligned}
$$

where the final simplification comes from retaining only terms with the largest number of scale factors. With the same approximations we have,

$$
\mathbf{R}_{\mu \nu} \longrightarrow-(D-1) \widehat{\mathbf{g}}_{\mu \nu} \widehat{\mathbf{g}}^{00} \mathbf{H}^{2} \mathbf{a}^{2}=-(D-1) \mathbf{H}^{2} \widehat{\mathbf{g}}^{00} \times \mathbf{g}_{\mu \nu} .
$$

This is the Einstein equation with a time-dependent cosmological constant,

$$
\boldsymbol{\Lambda}(x)=(D-1) \mathbf{H}^{2}(x) \times-\widehat{\mathbf{g}}^{00}(x) .
$$

So invoking the Transformation Ansatz does not avoid the reality of back-reaction.

\section{Epilogue}

The conventional way of thinking about inflationary back-reaction is that inflation continually rips long wavelength gravitons out of the vacuum and the self-gravitation between them slows the expansion rate by an ever-increasing amount as more and more of these gravitons come into causal contact $[38,82]$. One objection is that the Heisenberg field equations imply the Ricci scalar is constant $R=D \Lambda$ [47, 48]. However, the Ricci scalar and any other nonlinear field operator - diverges when acting on physical states, hence one should order it so as to extract the vacuum energy, the same way one does for $L_{0}$ in the Virasoro algebra of free string theory [87]. When that is done, back-reaction manifests as diagrams which make secular contributions to the vacuum energy.

Although the diagrams which contribute to secular back-reaction are completely causal in spacetime, they do derive from Fourier components of the graviton field operator whose physical wavelengths (in the background geometry) exceed the instantaneous Hubble radius. This occasions intense scepticism [59, 60], and has led to assertions that superhorizon modes are not accessible to a local observer, but would instead be subsumed into 
a transformation of his coordinate system [61-64]. The argument then runs that secular back-reaction is impossible because the local observer's metric does not even possess any of the super-horizon modes which might cause it.

We refer to this belief as the Transformation Ansatz and we showed in section 2 that the local observer's metric (2.2) it implies is properly a nonlocal field redefinition of the original metric. Secular back-reaction still occurs because the transformation which absorbs the super-horizon gravitons changes the conformal time coordinate upon which the scale factor depends. In section 3 we constructed the expansion rate (3.2) and field equation (3.25) which would be perceived by a local observer. Both of these quantities are operators so one can only discuss their statistical properties. With one assumption we were able to evaluate their expectation values (3.20), which show secular slowing at exactly the same order and with the same time dependence that is predicted in the conventional picture [82]. We conclude that the Transformation Ansatz does not preclude but rather confirms secular back-reaction.

The assumption we made to derive (3.20) is a large one: that there are no secular contributions at order $\kappa^{4}$ from the 1-point, 2-point and 3-point diagrams of figure 1 . We doubt that this can be correct. However, even if the other diagrams change the result (3.20), the fact remains that the local observer's expansion rate $\mathbf{H}(x)$ is both dynamical and timedependent. Note that $\mathbf{H}(x)=H / N(x)$, where $N(x)$ is the ADM lapse of the super-horizon gravitons, so one interpretation of secular back-reaction is that the continual freezing-in of modes gradually increases the time scale.

It seems to us that adopting the Transformation Ansatz is problematic because it denies the locality of interactions, and because it makes the number of degrees of freedom depend upon the background geometry and vary with time. However, we have been careful not to pronounce on the validity of the Transformation Ansatz; our point is merely that adopting it leads to secular back-reaction of the same sort that is predicted to occur when the super-horizon modes are retained. There seem to be complementary pictures:

- One can either employ the full metric - including super-horizon modes - and then one sees a vacuum energy whose time dependence derives from more and more modes coming into interaction; or

- One can excise the super-horizon modes, and then one sees a time-dependent expansion rate from a gradual increase in the lapse which sets the scale of time.

Although we are dubious as to the validity of the Transformation Ansatz there is no doubt that local observers couple only weakly to individual super-horizon modes. Hence, it may not be a bad approximation to assume that local observers perceive the geometry of the cleansed metric (2.2). Perhaps there is a sort of spacetime uncertainty principle at work: one could indeed infer the curvature by measuring the geodesic separation between freely falling observers, but resolving the contributions from modes of longer and longer wavelengths requires longer and longer times. So the result obtained within a Hubble time is the curvature of the infrared-cleansed metric (2.2). 
Finally, we would like to suggest that the increase in the gravitational lapse associated with the horizon crossing of a graviton mode can be viewed as a cosmological analogue of the famous gravitational "memory effect" [88-91]. Recall that the memory effect is a permanent shift in the geodesic separation between freely falling observers who experience the passage of a gravitational wave. The curvature is zero before and after the wave, yet the shift in their locations is real. Note that the small curvature associated with super-horizon gravitons is one of the chief arguments against them having any effect during inflation, and this very same argument could be invoked to deny the reality of the memory effect due to gravitational waves. That argument was wrong in flat space background and there is no reason to take it any more seriously during inflation.

The analogy with the memory effect is worth pursuing a little further. It has been shown [92] that the positional offset induced by the passage of a gravitational wave can be expressed as the action of a BMS transformation [93, 94], a class of diffeomorphisms which does not go to zero at spatial infinity. The cosmological analogue of these transformations has been constructed [95] and their action has been shown to add a super-horizon graviton [96]. The effect which interests us is not this linear one but rather a higher order part of what is the same transformation, so it is good to know that the full nonlinear extension exists. We should also note that the ability to absorb super-horizon gravitons using these infinite range diffeomorphisms was previously invoked by those who dispute the reality of secular graviton effects [52]. It will be seen that the very same argument could be used to deny the reality of gravitational memory and is therefore falsified.

\section{Acknowledgments}

This work was partially supported by the European Union's Seventh Framework Programme (FP7-REGPOT-2012-2013-1) under grant agreement number 316165; by the European Union's Horizon 2020 Programme under grant agreement 669288-SM-GRAV-ERC2014-ADG; by NSF grant PHY-1506513; and by the Institute for Fundamental Theory at the University of Florida.

Open Access. This article is distributed under the terms of the Creative Commons Attribution License (CC-BY 4.0), which permits any use, distribution and reproduction in any medium, provided the original author(s) and source are credited.

\section{References}

[1] V.K. Onemli and R.P. Woodard, Superacceleration from massless, minimally coupled $\phi^{4}$, Class. Quant. Grav. 19 (2002) 4607 [gr-qc/0204065] [inSPIRE].

[2] T. Prokopec, O. Tornkvist and R.P. Woodard, One loop vacuum polarization in a locally de Sitter background, Annals Phys. 303 (2003) 251 [gr-qc/0205130] [INSPIRE].

[3] T. Prokopec and R.P. Woodard, Production of massless fermions during inflation, JHEP 10 (2003) 059 [astro-ph/0309593] [INSPIRE].

[4] T. Brunier, V.K. Onemli and R.P. Woodard, Two loop scalar self-mass during inflation, Class. Quant. Grav. 22 (2005) 59 [gr-qc/0408080] [INSPIRE]. 
[5] S.-P. Miao and R.P. Woodard, Leading log solution for inflationary Yukawa, Phys. Rev. D 74 (2006) 044019 [gr-qc/0602110] [INSPIRE].

[6] T. Prokopec, N.C. Tsamis and R.P. Woodard, Two Loop Scalar Bilinears for Inflationary SQED, Class. Quant. Grav. 24 (2007) 201 [gr-qc/0607094] [INSPIRE].

[7] T. Prokopec, N.C. Tsamis and R.P. Woodard, Two loop stress-energy tensor for inflationary scalar electrodynamics, Phys. Rev. D 78 (2008) 043523 [arXiv:0802.3673] [INSPIRE].

[8] T.M. Janssen, S.P. Miao, T. Prokopec and R.P. Woodard, The Hubble Effective Potential, JCAP 05 (2009) 003 [arXiv:0904.1151] [InSPIRE].

[9] E.O. Kahya, V.K. Onemli and R.P. Woodard, A Completely Regular Quantum Stress Tensor with $w<-1$, Phys. Rev. D 81 (2010) 023508 [arXiv:0904.4811] [INSPIRE].

[10] E.O. Kahya, V.K. Onemli and R.P. Woodard, The Zeta-Zeta Correlator Is Time Dependent, Phys. Lett. B 694 (2011) 101 [arXiv: 1006.3999] [InSPIRE].

[11] H. Kitamoto and Y. Kitazawa, Non-linear $\sigma$-model in de Sitter space, Phys. Rev. D 83 (2011) 104043 [arXiv: 1012.5930] [INSPIRE].

[12] S. Park and R.P. Woodard, Scalar Contribution to the Graviton Self-Energy during Inflation, Phys. Rev. D 83 (2011) 084049 [arXiv:1101.5804] [inSPIRE].

[13] H. Kitamoto and Y. Kitazawa, Infra-red effects of Non-linear $\sigma$-model in de Sitter space, Phys. Rev. D 85 (2012) 044062 [arXiv:1109.4892] [INSPIRE].

[14] N.C. Tsamis and R.P. Woodard, The quantum gravitational back reaction on inflation, Annals Phys. 253 (1997) 1 [hep-ph/9602316] [INSPIRE].

[15] N.C. Tsamis and R.P. Woodard, One loop graviton selfenergy in a locally de Sitter background, Phys. Rev. D 54 (1996) 2621 [hep-ph/9602317] [INSPIRE].

[16] S.-P. Miao and R.P. Woodard, The fermion self-energy during inflation, Class. Quant. Grav. 23 (2006) 1721 [gr-qc/0511140] [INSPIRE].

[17] E.O. Kahya and R.P. Woodard, Quantum Gravity Corrections to the One Loop Scalar Self-Mass during Inflation, Phys. Rev. D 76 (2007) 124005 [arXiv:0709.0536] [INSPIRE].

[18] H. Kitamoto and Y. Kitazawa, Soft Gravitons Screen Couplings in de Sitter Space, Phys. Rev. D 87 (2013) 124007 [arXiv: 1203.0391] [INSPIRE].

[19] H. Kitamoto and Y. Kitazawa, Soft Graviton effects on Gauge theories in de Sitter Space, Phys. Rev. D 87 (2013) 124004 [arXiv: 1204.2876] [inSPIRE].

[20] S.P. Miao, Quantum Gravitational Effects on Massive Fermions during Inflation I, Phys. Rev. D 86 (2012) 104051 [arXiv:1207.5241] [INSPIRE].

[21] K.E. Leonard and R.P. Woodard, Graviton Corrections to Vacuum Polarization during Inflation, Class. Quant. Grav. 31 (2014) 015010 [arXiv:1304.7265] [INSPIRE].

[22] S. Boran, E.O. Kahya and S. Park, Quantum gravity corrections to the conformally coupled scalar self-mass-squared on de Sitter background, Phys. Rev. D 90 (2014) 124054 [arXiv: 1409.7753] [INSPIRE].

[23] D. Glavan, S.P. Miao, T. Prokopec and R.P. Woodard, Graviton Loop Corrections to Vacuum Polarization in de Sitter in a General Covariant Gauge, Class. Quant. Grav. 32 (2015) 195014 [arXiv:1504.00894] [INSPIRE]. 
[24] T. Prokopec, O. Tornkvist and R.P. Woodard, Photon mass from inflation, Phys. Rev. Lett. 89 (2002) 101301 [astro-ph/0205331] [INSPIRE].

[25] T. Prokopec and R.P. Woodard, Dynamics of superhorizon photons during inflation with vacuum polarization, Annals Phys. 312 (2004) 1 [gr-qc/0310056] [INSPIRE].

[26] B. Garbrecht and T. Prokopec, Fermion mass generation in de Sitter space, Phys. Rev. D 73 (2006) 064036 [gr-qc/0602011] [InSPIRE].

[27] S.P. Miao and R.P. Woodard, Gravitons Enhance Fermions during Inflation, Phys. Rev. D 74 (2006) 024021 [gr-qc/0603135] [INSPIRE].

[28] E.O. Kahya and V.K. Onemli, Quantum Stability of a $w<-1$ Phase of Cosmic Acceleration, Phys. Rev. D 76 (2007) 043512 [gr-qc/0612026] [INSPIRE].

[29] E.O. Kahya and R.P. Woodard, Scalar field equations from quantum gravity during inflation, Phys. Rev. D 77 (2008) 084012 [arXiv:0710.5282] [INSPIRE].

[30] P.J. Mora, N.C. Tsamis and R.P. Woodard, Hartree approximation to the one loop quantum gravitationalcorrection to the graviton mode function on de Sitter, JCAP 10 (2013) 018 [arXiv: 1307.1422] [INSPIRE].

[31] C.L. Wang and R.P. Woodard, Excitation of Photons by Inflationary Gravitons, Phys. Rev. D 91 (2015) 124054 [arXiv:1408.1448] [INSPIRE].

[32] D. Glavan, S.P. Miao, T. Prokopec and R.P. Woodard, One loop graviton corrections to dynamical photons in de Sitter, Class. Quant. Grav. 34 (2017) 085002 [arXiv:1609.00386] [INSPIRE].

[33] H. Degueldre and R.P. Woodard, One Loop Field Strengths of Charges and Dipoles on a Locally de Sitter Background, Eur. Phys. J. C 73 (2013) 2457 [arXiv:1303.3042] [INSPIRE].

[34] D. Glavan, S.P. Miao, T. Prokopec and R.P. Woodard, Electrodynamic Effects of Inflationary Gravitons, Class. Quant. Grav. 31 (2014) 175002 [arXiv:1308.3453] [INSPIRE].

[35] S. Park, T. Prokopec and R.P. Woodard, Quantum Scalar Corrections to the Gravitational Potentials on de Sitter Background, JHEP 01 (2016) 074 [arXiv:1510.03352] [INSPIRE].

[36] S. Weinberg, Quantum contributions to cosmological correlations, Phys. Rev. D 72 (2005) 043514 [hep-th/0506236] [INSPIRE].

[37] S. Weinberg, Quantum contributions to cosmological correlations. II. Can these corrections become large?, Phys. Rev. D 74 (2006) 023508 [hep-th/0605244] [INSPIRE].

[38] N.C. Tsamis and R.P. Woodard, Quantum gravity slows inflation, Nucl. Phys. B 474 (1996) 235 [hep-ph/9602315] [INSPIRE].

[39] V.K. Onemli and R.P. Woodard, Quantum effects can render $w<-1$ on cosmological scales, Phys. Rev. D 70 (2004) 107301 [gr-qc/0406098] [inSPIRE].

[40] T. Prokopec, N.C. Tsamis and R.P. Woodard, Stochastic Inflationary Scalar Electrodynamics, Annals Phys. 323 (2008) 1324 [arXiv:0707.0847] [INSPIRE].

[41] A.A. Starobinsky, Stochastic de Sitter (inflationary) Stage In The Early Universe, Lect. Notes Phys. 246 (1986) 107 [InSPIRE].

[42] A.A. Starobinsky and J. Yokoyama, Equilibrium state of a selfinteracting scalar field in the de Sitter background, Phys. Rev. D 50 (1994) 6357 [astro-ph/9407016] [INSPIRE]. 
[43] R.P. Woodard, A leading logarithm approximation for inflationary quantum field theory, Nucl. Phys. Proc. Suppl. 148 (2005) 108 [astro-ph/0502556] [InSPIRE].

[44] N.C. Tsamis and R.P. Woodard, Stochastic quantum gravitational inflation, Nucl. Phys. B 724 (2005) 295 [gr-qc/0505115] [INSPIRE].

[45] F. Finelli, G. Marozzi, A.A. Starobinsky, G.P. Vacca and G. Venturi, Generation of fluctuations during inflation: Comparison of stochastic and field-theoretic approaches, Phys. Rev. D 79 (2009) 044007 [arXiv: 0808.1786] [INSPIRE].

[46] F. Finelli, G. Marozzi, A.A. Starobinsky, G.P. Vacca and G. Venturi, Stochastic growth of quantum fluctuations during slow-roll inflation, Phys. Rev. D 82 (2010) 064020 [arXiv: 1003.1327] [INSPIRE].

[47] J. Garriga and T. Tanaka, Can infrared gravitons screen Lambda?, Phys. Rev. D 77 (2008) 024021 [arXiv:0706.0295] [INSPIRE].

[48] N.C. Tsamis and R.P. Woodard, Comment on 'Can infrared gravitons screen Lambda?', Phys. Rev. D 78 (2008) 028501 [arXiv:0708.2004] [INSPIRE].

[49] A. Vilenkin and L.H. Ford, Gravitational Effects upon Cosmological Phase Transitions, Phys. Rev. D 26 (1982) 1231.

[50] A.D. Linde, Scalar Field Fluctuations in Expanding Universe and the New Inflationary Universe Scenario, Phys. Lett. B 116 (1982) 335 [INSPIRE].

[51] A.A. Starobinsky, Dynamics of Phase Transition in the New Inflationary Universe Scenario and Generation of Perturbations, Phys. Lett. B 117 (1982) 175 [INSPIRE].

[52] A. Higuchi, D. Marolf and I.A. Morrison, de Sitter invariance of the dS graviton vacuum, Class. Quant. Grav. 28 (2011) 245012 [arXiv:1107.2712] [INSPIRE].

[53] S.P. Miao, N.C. Tsamis and R.P. Woodard, Gauging away Physics, Class. Quant. Grav. 28 (2011) 245013 [arXiv: 1107.4733] [INSPIRE].

[54] I.A. Morrison, On cosmic hair and "de Sitter breaking" in linearized quantum gravity, arXiv:1302.1860 [INSPIRE].

[55] S.P. Miao, P.J. Mora, N.C. Tsamis and R.P. Woodard, Perils of analytic continuation, Phys. Rev. D 89 (2014) 104004 [arXiv:1306.5410] [INSPIRE].

[56] M.B. Fröb, The Weyl tensor correlator in cosmological spacetimes, JCAP 12 (2014) 010 [arXiv: 1409.7964] [INSPIRE].

[57] R.P. Woodard, Some Inconvenient Truths, JHEP 05 (2016) 152 [arXiv:1506.04252] [INSPIRE].

[58] S.P. Miao and R.P. Woodard, Issues Concerning Loop Corrections to the Primordial Power Spectra, JCAP 07 (2012) 008 [arXiv:1204.1784] [INSPIRE].

[59] Y. Urakawa and T. Tanaka, Influence on Observation from IR Divergence during Inflation. I, Prog. Theor. Phys. 122 (2009) 779 [arXiv:0902.3209] [INSPIRE].

[60] Y. Urakawa and T. Tanaka, Influence on observation from IR divergence during inflation: Multi field inflation, Prog. Theor. Phys. 122 (2010) 1207 [arXiv: 0904.4415] [INSPIRE].

[61] S.B. Giddings and M.S. Sloth, Semiclassical relations and IR effects in de Sitter and slow-roll space-times, JCAP 01 (2011) 023 [arXiv: 1005.1056] [INSPIRE]. 
[62] Y. Urakawa and T. Tanaka, IR divergence does not affect the gauge-invariant curvature perturbation, Phys. Rev. D 82 (2010) 121301 [arXiv:1007.0468] [inSPIRE].

[63] T. Tanaka and Y. Urakawa, Dominance of gauge artifact in the consistency relation for the primordial bispectrum, JCAP 05 (2011) 014 [arXiv:1103.1251] [INSPIRE].

[64] S.B. Giddings and M.S. Sloth, Cosmological observables, IR growth of fluctuations and scale-dependent anisotropies, Phys. Rev. D 84 (2011) 063528 [arXiv:1104.0002] [INSPIRE].

[65] S. Basu and R.P. Woodard, Testing an Ansatz for the Leading Secular Loop Corrections from Quantum Gravity during Inflation, Class. Quant. Grav. 33 (2016) 205007 [arXiv: 1606.02417] [INSPIRE].

[66] L. Senatore and M. Zaldarriaga, On Loops in Inflation II: IR Effects in Single Clock Inflation, JHEP 01 (2013) 109 [arXiv:1203.6354] [INSPIRE].

[67] L. Senatore and M. Zaldarriaga, A Note on the Consistency Condition of Primordial Fluctuations, JCAP 08 (2012) 001 [arXiv:1203.6884] [INSPIRE].

[68] G.L. Pimentel, L. Senatore and M. Zaldarriaga, On Loops in Inflation III: Time Independence of zeta in Single Clock Inflation, JHEP 07 (2012) 166 [arXiv:1203.6651] [INSPIRE].

[69] T. Tanaka and Y. Urakawa, Strong restriction on inflationary vacua from the local gauge invariance I: Local gauge invariance and infrared regularity, PTEP 2013 (2013) $083 \mathrm{E} 01$ [arXiv: 1209.1914] [INSPIRE].

[70] T. Tanaka and Y. Urakawa, Strong restriction on inflationary vacua from the local gauge invariance II: Infrared regularity and absence of secular growth in the Euclidean vacuum, PTEP 2013 (2013) 063E02 [arXiv:1301.3088] [INSPIRE].

[71] T. Tanaka and Y. Urakawa, Strong restriction on inflationary vacua from the local gauge invariance III: Infrared regularity of graviton loops, PTEP 2014 (2014) 073E01 [arXiv: 1402.2076] [INSPIRE].

[72] T. Tanaka and Y. Urakawa, Conservation of $\zeta$ with radiative corrections from heavy field, JCAP 06 (2016) 020 [arXiv:1510.05059] [INSPIRE].

[73] R.P. Woodard, The Vierbein Is Irrelevant in Perturbation Theory, Phys. Lett. B 148 (1984) 440 [INSPIRE].

[74] R. Arnowitt and S. Deser, Quantum Theory of Gravitation: General Formulation and Linearized Theory, Phys. Rev. 113 (1959) 745.

[75] R.L. Arnowitt, S. Deser and C.W. Misner, Canonical variables for general relativity, Phys. Rev. 117 (1960) 1595.

[76] R.L. Arnowitt, S. Deser and C.W. Misner, The dynamics of general relativity, Gen. Rel. Grav. 40 (2008) 1997 [gr-qc/0405109] [INSPIRE].

[77] N.C. Tsamis and R.P. Woodard, The physical basis for infrared divergences in inflationary quantum gravity, Class. Quant. Grav. 11 (1994) 2969 [INSPIRE].

[78] S.-P. Miao and R.P. Woodard, A Simple Operator Check of the Effective Fermion Mode Function during Inflation, Class. Quant. Grav. 25 (2008) 145009 [arXiv:0803.2377] [INSPIRE].

[79] N.C. Tsamis and R.P. Woodard, The structure of perturbative quantum gravity on a de Sitter background, Commun. Math. Phys. 162 (1994) 217 [INSPIRE]. 
[80] R.P. Woodard, de Sitter breaking in field theory, gr-qc/0408002 [INSPIRE].

[81] N.C. Tsamis and R.P. Woodard, Dimensionally regulated graviton 1-point function in de Sitter, Annals Phys. 321 (2006) 875 [gr-qc/0506056] [INSPIRE].

[82] N.C. Tsamis and R.P. Woodard, A Gravitational Mechanism for Cosmological Screening, Int. J. Mod. Phys. D 20 (2011) 2847 [arXiv:1103.5134] [INSPIRE].

[83] B. Allen and A. Folacci, The Massless Minimally Coupled Scalar Field in De Sitter Space, Phys. Rev. D 35 (1987) 3771.

[84] E. Lifshitz, Republication of: On the gravitational stability of the expanding universe, J. Phys. (USSR) 10 (1946) 116 [INSPIRE].

[85] Y. Aharonov and D. Bohm, Significance of electromagnetic potentials in the quantum theory, Phys. Rev. 115 (1959) 485.

[86] S.P. Miao, N.C. Tsamis and R.P. Woodard, Invariant measure of the one-loop quantum gravitational backreaction on inflation, Phys. Rev. D 95 (2017) 125008 [arXiv:1702.05694] [INSPIRE].

[87] P. Goddard and C.B. Thorn, Compatibility of the Dual Pomeron with Unitarity and the Absence of Ghosts in the Dual Resonance Model, Phys. Lett. B 40 (1972) 235 [InSPIRE].

[88] Ya.B. Zel'dovich and A.G. Polnarev, Radiation of gravitational waves by a cluster of superdense stars, Sov. Astron. 18 (1974) 17.

[89] V.B. Braginsky and L.P. Grishchuk, Kinematic Resonance and Memory Effect in Free Mass Gravitational Antennas, Sov. Phys. JETP 62 (1985) 427 [InSPIRE].

[90] M. Ludvigsen, Geodesic deviation at null infinity and the physical effects of very long wave gravitational radiation, Gen. Rel. Grav. 21 (1989) 1205 [INSPIRE].

[91] D. Christodoulou, Nonlinear nature of gravitation and gravitational wave experiments, Phys. Rev. Lett. 67 (1991) 1486 [INSPIRE].

[92] A. Strominger and A. Zhiboedov, Gravitational Memory, BMS Supertranslations and Soft Theorems, JHEP 01 (2016) 086 [arXiv:1411.5745] [INSPIRE].

[93] H. Bondi, M.G.J. van der Burg and A.W.K. Metzner, Gravitational waves in general relativity. 7. Waves from axisymmetric isolated systems, Proc. Roy. Soc. Lond. A 269 (1962) 21 [INSPIRE].

[94] R.K. Sachs, Gravitational waves in general relativity. 8. Waves in asymptotically flat space-times, Proc. Roy. Soc. Lond. A 270 (1962) 103 [InSPIRE].

[95] K. Hinterbichler, L. Hui and J. Khoury, An Infinite Set of Ward Identities for Adiabatic Modes in Cosmology, JCAP 01 (2014) 039 [arXiv: 1304.5527] [INSPIRE].

[96] R.Z. Ferreira, M. Sandora and M.S. Sloth, Asymptotic Symmetries in de Sitter and Inflationary Spacetimes, JCAP 04 (2017) 033 [arXiv: 1609.06318] [INSPIRE]. 